ERRATA

\title{
Isolation and characterization of a novel gene $C L U A P 1$ whose expression is frequently upregulated in colon cancer
}

Meiko Takahashi ${ }^{1}$, Yu-Min Lin², Yusuke Nakamura ${ }^{1}$ and Yoichi Furukawa ${ }^{1}$

Oncogene (2005) 24, 4320. doi:10.1038/sj.onc.1208763

Correction to: Oncogene (2004) 23, 9289-9294. doi:10.1038/sj.onc. 1208100

Published online 11 October 2004
Owing to a typesetting error, Figure 2 of the above paper was published incorrectly. The correct version of the figure is given here.

a

\section{The PI 3-kinase/Akt signaling pathway is activated due to aberrant Pten expression and targets transcription factors NF- $\kappa \mathrm{B}$ and c-Myc in pancreatic cancer cells}

Takayuki Asano, Yixin Yao, Jijiang Zhu, Donghui Li, James L Abbruzzese and Shrikanth AG Reddy

Oncogene (2005) 24, 4320. doi:10.1038/sj.onc.1208766

Correction to: Oncogene (2004) 23, 8571-8580.

doi: $10.1038 /$ sj.onc. 1207902

Due to a typesetting error, Figure 6c of this paper contained an incorrect legend. The figure with the correct legend is given here.

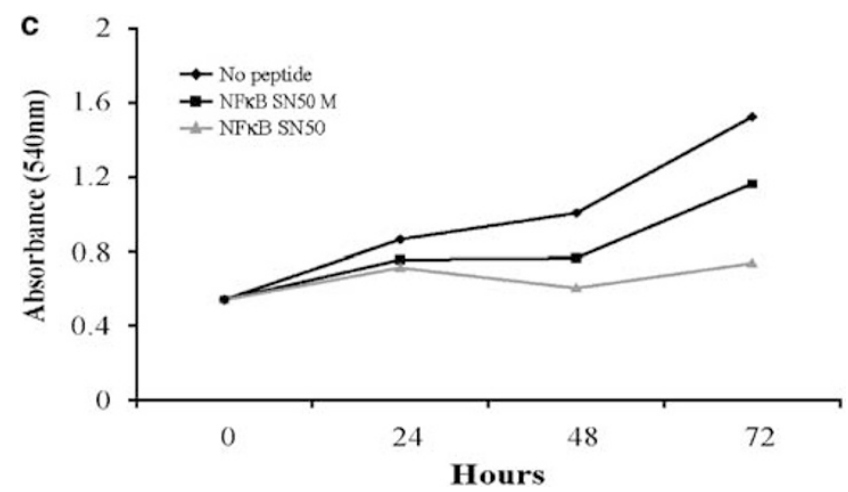

